\title{
dbOGAP - An Integrated Bioinformatics Resource for Protein O-GlcNAcylation
}

\author{
Jinlian Wang ${ }^{1}$, Manabu Torii ${ }^{2}$, Hongfang Liu ${ }^{3}$, Gerald W Hart ${ }^{4}$ and Zhang-Zhi Hu${ }^{{ }^{*}}$
}

\begin{abstract}
Background: Protein O-GlcNAcylation (or O-GICNAc-ylation) is an O-linked glycosylation involving the transfer of $\beta$ $\mathrm{N}$-acetylglucosamine to the hydroxyl group of serine or threonine residues of proteins. Growing evidences suggest that protein O-GlcNAcylation is common and is analogous to phosphorylation in modulating broad ranges of biological processes. However, compared to phosphorylation, the amount of protein O-GlcNAcylation data is relatively limited and its annotation in databases is scarce. Furthermore, a bioinformatics resource for OGlcNAcylation is lacking, and an O-GlcNAcylation site prediction tool is much needed.

Description: We developed a database of O-GlcNAcylated proteins and sites, dbOGAP, primarily based on literature published since O-GlcNAcylation was first described in 1984. The database currently contains $\sim 800$ proteins with experimental O-GlcNAcylation information, of which $\sim 61 \%$ are of humans, and 172 proteins have a total of 400 O-GlcNAcylation sites identified. The O-GlcNAcylated proteins are primarily nucleocytoplasmic, including membrane- and non-membrane bounded organelle-associated proteins. The known O-GlcNAcylated proteins exert a broad range of functions including transcriptional regulation, macromolecular complex assembly, intracellular transport, translation, and regulation of cell growth or death. The database also contains $\sim 365$ potential O-GlcNAcylated proteins inferred from known O-GlcNAcylated orthologs. Additional annotations, including other protein posttranslational modifications, biological pathways and disease information are integrated into the database. We developed an O-GlcNAcylation site prediction system, OGlcNAcScan, based on Support Vector Machine and trained using protein sequences with known O-GlcNAcylation sites from dbOGAP. The site prediction system achieved an area under ROC curve of $74.3 \%$ in five-fold cross-validation. The dbOGAP website was developed to allow for performing search and query on O-GlcNAcylated proteins and associated literature, as well as for browsing by gene names, organisms or pathways, and downloading of the database. Also available from the website, the OGICNAcScan tool presents a list of predicted O-GIcNAcylation sites for given protein sequences.

Conclusions: dbOGAP is the first public bioinformatics resource to allow systematic access to the O-GlcNAcylated proteins, and related functional information and bibliography, as well as to an O-GlcNAcylation site prediction tool. The resource will facilitate research on O-GlcNAcylation and its proteomic identification.
\end{abstract}

\section{Background}

O-GlcNAcylation, or O-GlcNAc-ylation to distinguish it from acylation, is an O-linked glycosylation involving the $\beta$-attachment of a single $\mathrm{N}$-acetylglucosamine (GlcNAc) to the serine (Ser)/threonine (Thr) residues catalyzed by O-GlcNAc transferase (OGT), whose removal is catalyzed by O-GlcNAcase (OGA) [1]. The two O-GlcNAc cycling enzymes OGT and OGA are each encoded by a

\footnotetext{
* Correspondence: zh9@georgetown.edu

'Department of Oncology, Georgetown University Medical Center, 3300

Whitehaven Street, Suite 1200, NW, Washington, DC 20007, USA

Full list of author information is available at the end of the article
}

single gene in mammalian species. Unlike N-linked or mucin-type O-linked glycosylation, O-GlcNAcylation occurs primarily in nucleocytoplasmic proteins [1]. Analogous to phosphorylation, the modification is dynamic and the O-GlcNAc moiety is not further extended [1]. OGlcNAcylation is also often reciprocal to phosphorylation at the same or adjacent Ser/Thr residues [1-3], which led to a "Yin-Yang" hypothesis on protein functions modulated by the two post-translational modifications (PTMs) [4] through competitively blocking each other's occupancy at given sites. For example, reciprocal O-GlcNAcylation and phosphorylation at the same Ser16 of murine 
estrogen receptor $\beta$ (ER $\beta$ modulate the degradation of ER $\beta$ by stabilizing or destabilizing the protein, respectively [5]. Similarly, O-GlcNAcylation of p53 at Ser149 is associated with decreased phosphorylation at the adjacent Thr155, resulting in decreased p53 ubiquitination and subsequent degradation, thus stabilizing p53 [6]. In contrast to the enormous body of research on phosphorylation, the amount of research on O-GlcNAcylation has been disproportionally small due to difficulties in detecting the O-GlcNAc group, partly because of its being labile, dynamic, and substoichiometric [7]. Over 600 proteins have been reported to be O-GlcNAcylated since it was first identified in 1984 [8], many of which were identified in recent years [1-3,9-11] as a result of improved mass spectrometry technologies. Growing evidences now suggest that O-GlcNAcylation is very common and has broad roles in physiology and diseases, especially through its reciprocal interplay with phosphorylation, e.g., regulation of insulin signaling, transcription, and roles in diabetes and neurodegenerative diseases [2].

A number of bioinformatics databases have been developed for protein post-translational modifications, including those of general PTMs, e.g., dbPTM [12], or specific types, e.g., databases of protein phosphorylation, e.g., PhosphoELM [13], PhosphoSite [14], and those of protein glycosylation [15], ubiquitination [16] and protease cleavage [17]. By contrast, there has been no special database dedicated to O-GlcNAcylated proteins and sites, and their annotations are also scarce in protein databases, e.g., only 100 experimental O-GlcNAcylation sites for 35 proteins are currently annotated in UniProtKB [18]. Moreover, O-GlcNAcylation annotations have not been included in the specialized glycosylation databases (e.g., GlycoBase, the Functional Glycomics Gateway) [15,19].

Because of growing interests in studying the crucial roles of O-GlcNAcylation in cell signaling and many other cellular processes, identifying the site motifs and computationally predicting the O-GlcNAcylation sites become important bioinformatics tasks to assist those studies. Unlike N-linked glcycosylation with a consensus motif of "Asn-X-Thr/Ser", O-linked glycosylation, including mucin-type O-glycosylation and O-GlcNAc glycosylation, has not yet found well-defined sequence motifs. The past effort in developing prediction method for O-glycosylation has mostly focused on the mucintype [20-23]. To our best knowledge there has been only one site prediction tool for O-GlcNAcylation, YinOYang, which is an artificial neural network system trained on sequence fragments of $\sim 40$ GlcNAcylation sites available at the time [24]. The motif of O-GlcNAcylation remains poorly defined, and there is a pressing need to develop an O-GlcNAcylation site prediction tool based on a much greater number of experimental O-GlcNAcylation sites available now.
Here we report the development of a database of $\underline{\mathrm{O}}-$ GlcNAcylated proteins and sites (dbOGAP) for all currently known O-GlcNAcylated proteins reported from literature, and of an O-GlcNAcylation site prediction system (OGlcNAcScan) based on nearly $400 \mathrm{O}$-GlcNAcylation sites. Both the database and the prediction system are available through the dbOGAP web site, which serves as a public bioinformatics resource to facilitate research on O-GlcNAcylated proteins and to assist proteomic identification of O-GlcNAcylation sites.

\section{Construction and Content}

\section{The Database Development}

The primary data source used for developing the dbOGAP database is literature about O-GlcNAcylated proteins published since O-GlcNAcylation was first discovered in early 1980's [8]. Figure 1 depicts the overall workflow of the dbOGAP database and web site development. About 500 original and review articles were retrieved from PubMed (April 2010) that are related to protein O-GlcNAcylation and/or the OGlcNAc cycling enzymes OGT and OGA. Abstracts and full-length articles were used to identify experimentally determined O-GlcNAcylated proteins and sites. The proteins were then mapped to UniProtKB entry records based on sequences and/or sequence identifiers (IDs) followed by manual verification. O-GlcNAcylated proteins and sites determined only from large-scale mass spectrometry (MS) without further validation using targeted MS and/or additional biochemical methods were annotated with evidence tags (e.g., "LS: MALDI-TOFMS"). Orthologs of known O-GlcNAcylated proteins with identified O-GlcNAcylation sites were populated based on the HomoloGene groups [25] and/or BLAST neighbors [26], where the potential O-GlcNAcylation sites on the orthologs were inferred based on the conserved Ser/Thr residues. The experimental or inferred O-GlcNAcylation was attributed with literature (PubMed ID) or inference (from orthologs), respectively. A small number of currently annotated O-GlcNAcylated proteins in UniProtKB were also integrated into dbOGAP with the source attributed. Additional protein annotations, including other protein modifications (e.g., phosphorylation) and site features, Gene Ontology, pathways and disease information were integrated into $\mathrm{dbO}$ GAP from UniProtKB [18] or iProClass [27] databases.

\section{The O-GIcNAc Site Prediction}

An O-GlcNAcylation site prediction system, OGlcNAcScan, was developed based on annotated O-GlcNAcylation sites in dbOGAP using the SVM ${ }^{\text {light }}$ implementation of Support Vector Machine (SVM) [28]. A training data set of the prediction system consists of 373 positive instances that are experimental O-GlcNAcylation sites in 167 


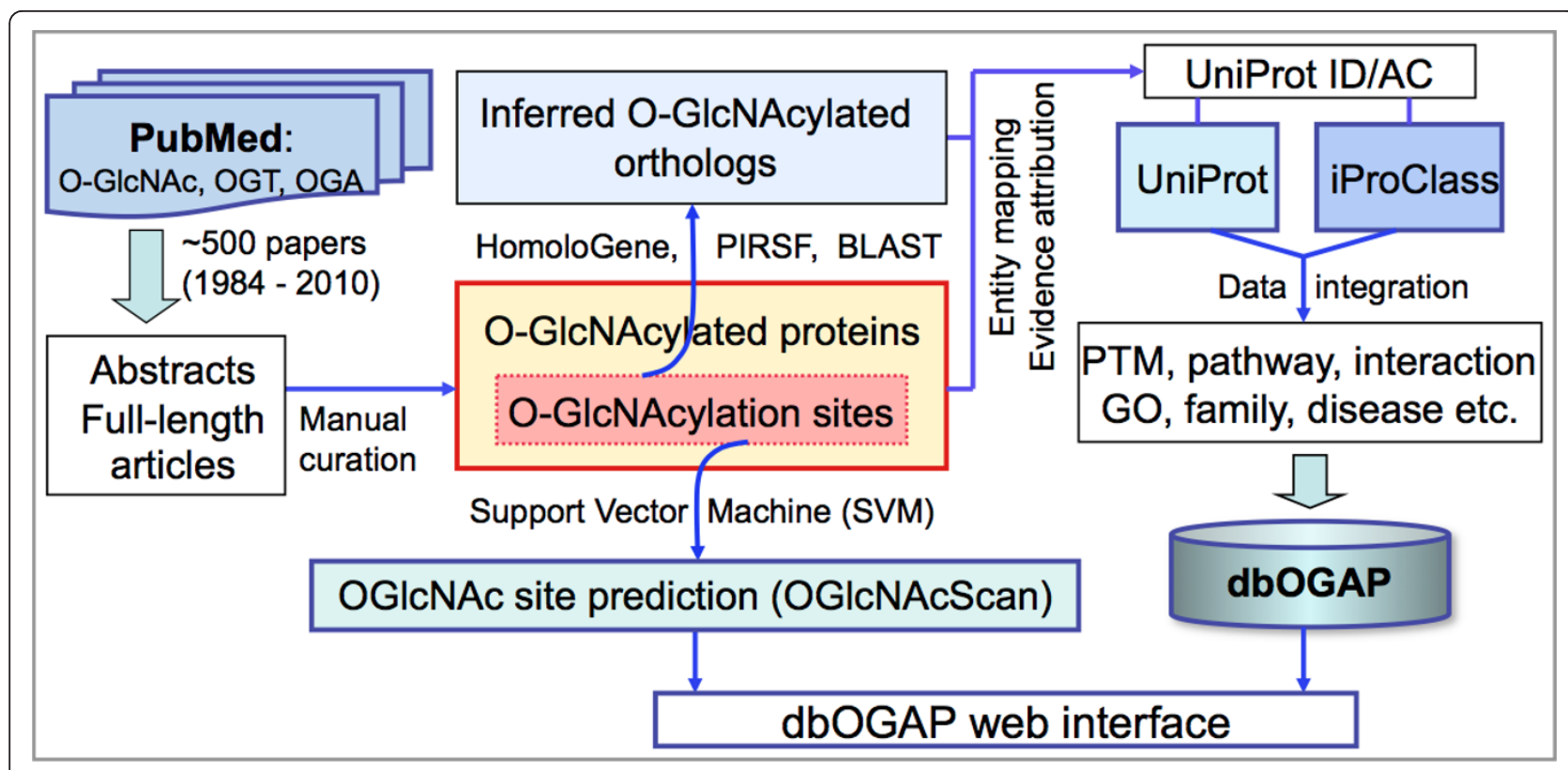

Figure 1 Overall workflow of dbOGAP development.

protein sequences from dbOGAP, and also of 29,897 negative instances that are the rest of the un-annotated Ser/ Thr sites in the same protein sequences. Given a Ser/Thr site, $n$ upstream and $n$ downstream amino acids were regarded as its sequence context and then $2 n+1$ amino acids, including the O-GlcNAcylated Ser or Thr residue in the middle, were converted into a vector of binary values (0 or 1 ) using the widely-used sparse encoding method described, for example, in Julenius et al. 2005 [21]. Note, if the site is less than $n$ amino acid away from the sequence terminals, the end-of-sequence symbol is padded at the terminal as many as needed to derive a fixed-length sequence fragment. In this encoding method, each amino acid type and the end-of-sequence symbol is coded with 21 binary values, e.g., 100...0 (one followed by 20 zeros) for Ala, $010 \ldots 0$ for Arg, ..., and $000 . . .1$ for end-of-sequence), and the resulting feature vector consists of $21 \times(2 n+1)$ binary values. For different values of $n$, we trained SVM classifiers with the RBF kernel. The parameters involving these classifiers, $C$ and $\gamma$, were optimized through 5 -fold cross-validation tests, where classifiers were trained and tested, respectively, on a four-fifths and the remaining one-fifth of the data set for five times. We explored different sequence encoding methods, such as frequencies of amino acid types [21,23] and gappy bi-grams/dimers [22], but the orthodox sparse encoding method with $n=5$ yielded the best prediction performance.

\section{The Database and the Web site Implementation}

The dbOGAP database is implemented using the open source relational database management system, MySQL, with tables to store and manage the O-GlcNAcylation protein entries, O-GlcNAcylation sites from different sources and related literature information. The database is deployed on RedHat Enterprise Linux operating system (version 5.5). The Apache web server (version 2.2.15) (http://httpd.apache.org/) with the security enhanced module ModSecurity (version 2.5.10, http:// www.modsecurity.org/), was deployed for the dbOGAP web site. All data query and retrieval from the dbOGAP web site is accomplished by scripts written in Perl, PHP and Javascript.

\section{Utility}

\section{The Database Contents}

The current version of dbOGAP contains 1163 entries, including 798 experimentally determined O-GlcNAcylated proteins (Figure 2, Left, A), and 365 proteins with inferred O-GlcNAcylation sites (total 735 sites) based on OGlcNAcylated orthologs (Figure 2, Left, D). About 22\% of all known O-GlcNAcylated proteins (172/798) have OGlcNAcylation sites identified (404 sites), among which 140 proteins also have known phosphorylation sites (1581 sites) as well as $357 \mathrm{O}$-GlcNAcylation sites (Figure 2, Left, $\mathrm{B}, \mathrm{C})$. Interestingly, 48 of those 140 proteins have $122 \mathrm{Ser} /$ Thr sites that are potentially Yin-Yang sites to be subjected to possible reciprocal regulation by O-GlcNAcylation and phosohorylation, including 42 identical sites and 74 non-identical sites that are within 4 amino acids away from each other (Table 1). Overall, the number of currently identified O-GlcNAcylation sites is only 11\% (404/ 3687 ) of that of phosphorylation sites on all known 

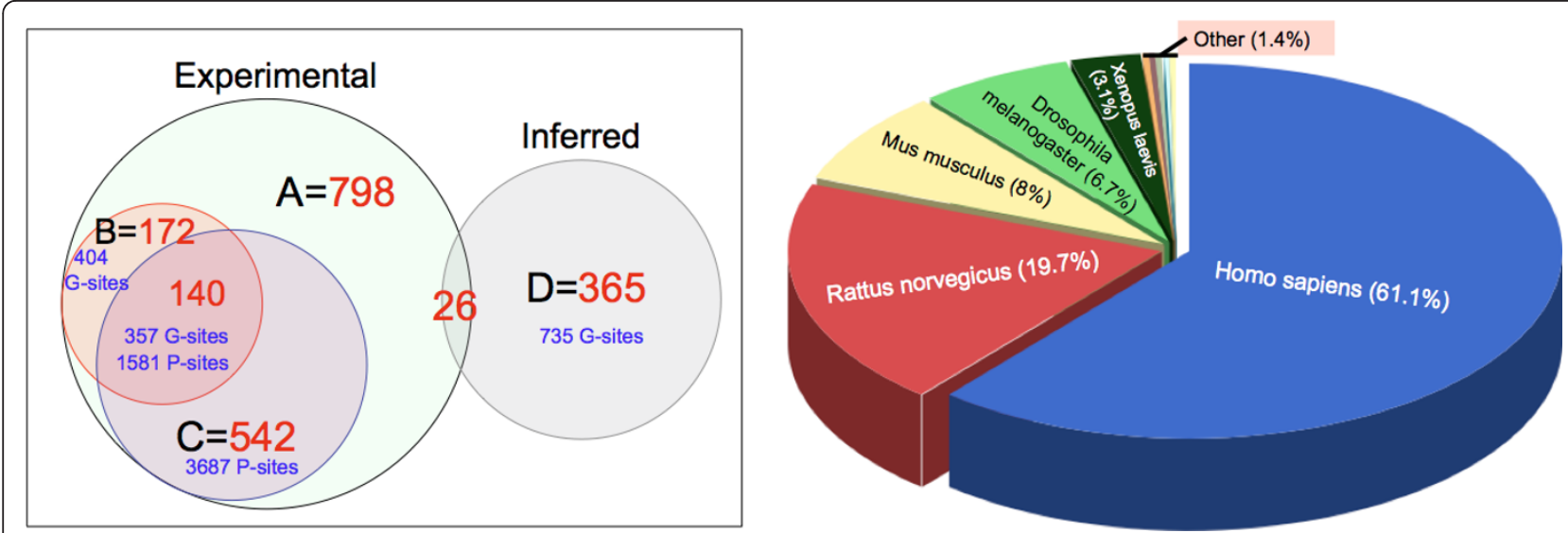

Figure 2 Statistics of protein entries in dbOGAP. Left, Venn diagram showing the number of O-GlcNAcylated proteins and modification sites in the dbOGAP database. There are a total of 1163 protein entries in the database. A - Experimental O-GlcNAcylated proteins; B - Proteins with identified O-GlcNAcylation sites (G-sites); C - Proteins with identified phosphorylation sites (P-sites); D - Proteins with inferred O-GlcNAcylation sites based on orthologs with known O-GlcNAcylation sites. Right, Taxonomic distributions of experimentally determined O-GlcNAcylated proteins in dbOGAP. Numbers shown in the pie chart are percentage of proteins in given species over the total number of proteins in the database. "Other" species include Bos taurus (3 protein entries), Gallus gallus (2), Coturnix coturnix japonica (1), Rhea americana (1), Macaca mulatta (1) and Viruses (3).

O-GlcNAcylated proteins. Further, among all experimentally determined O-GlcNAcylated proteins, most $(\sim 61 \%)$ are of humans, and other organisms include rat $(19.7 \%)$, mouse (8\%), fruit fly (6.7\%), and African frog (3.1\%) (Figure 2, Right).

\section{Functional profiles of O-GICNAcylated proteins}

We analyzed Gene Ontology (GO) profiles of currently known human O-GlcNAcylated proteins ( 490) using the DAVID tool [29]. We first examined the major enriched GO categories of O-GlcNAcylated proteins annotated with GO terms at higher levels of GO hierarchy (covering $\geq 10 \%$ of the proteins) (Table 2). As shown by the GO Cellular Components profiling, O-GlcNAcylated proteins are mostly those of nucleoplasmic distribution, including membrane or non-membrane bounded organelles, cytosol, cytoskeleton, and nuclear compartments. The O-GlcNAcylated proteins mainly possess nucleotide and nucleic acid binding activities and transcription regulator activities (GO Molecular Function), and participate in transcriptional regulation, macromolecular complex assembly, intracellular transport, translation, regulation of cell cycle and apoptosis, and regulation of macromolecule metabolic process (GO Biological Processes).

We further examined the O-GlcNAcylated proteins for enrichment of GO terms at deeper levels of the GO hierarchy. As summarized in [Additional file 1, Supplementary Table S1], the top enriched GO biological processes relate to protein translation, carbohydrate (glucose) metabolism, RNA processing/splicing, and RNA/protein transport, followed by macromolecular complex and organelle organization, regulation of cell cycle and cell death, chromosome organization and transcription, regulation of protein and other small molecule metabolisms. The enriched GO molecular functions include nucleoside, nucleotide and nucleic acid binding, transcription factor activity, protein binding and other molecular activities. The enriched GO cellular components include cytosol, organelle lumen and non-membrane-bounded organelles, nuclear compartments such as nucleoplasm, nuclear pore and nucleolus, ribosome and cytoskeleton, nuclear protein complexes and chromatin, membrane and vesicle associated spaces, and contractile associated proteins. Notably, although significant proportions of known O-GlcNAcylated proteins are associated with intracellular membranes or inner side of plasma membrane, only a few plasma transmembrane proteins, such as glucose transporters and notch receptor were reported to be O-GlcNAcylated [30-32]. Therefore O-GlcNAcylated proteins are primarily nucleocytoplasmic and are engaged in broad biological functions.

\section{Pathways and disease processes related to O-GICNAcylated proteins}

We examined pathway profiles of O-GlcNAcylated human proteins using GeneGO Pathway Maps [33]. A wide range of cellular pathways contain significant numbers of proteins that are known to be subjected to OGlcNAcylation, including pathways involved in growth, development and differentiation, immune and inflammatory responses, cytoskeleton remodeling, and metabolic pathways such as gluconeogenesis. A total of 141 annotated GeneGO pathways are significantly enriched for the O-GlcNAcylated human proteins (p-value $<1.0 \mathrm{E}$ $03)$. Table 3 lists 42 pathways that are enriched at a $\mathrm{p}$-value of $<1.0 \mathrm{E}-05$, many of which are growth factor 
Table 1 O-GIcNAcylation and phosphorylation occurring at identical or adjacent (+/- 4 amino acids) serine/threonine $(\mathrm{S} / \mathrm{T})$ sites of O-GIcNAcylated proteins

\begin{tabular}{|c|c|c|c|c|}
\hline UniProt ID & Gene name & O-GIcNAcylation site & Identical phosphorylation $\mathrm{S} / \mathrm{T}$ site & Adjacent phosphorylation $\mathrm{S} / \mathrm{T}$ site \\
\hline RRP1B_HUMAN & RRP1B & S731 & S731 & $\mathrm{T} 728, \mathrm{~S} 732, \mathrm{~S} 735$ \\
\hline CARF_HUMAN & CDKN2AIP & S348 & & T345 \\
\hline VIME_HUMAN & VIM & S7, T33, S34, S55 & S7, T33, S34, S55 & $\mathrm{S} 5, \mathrm{~S} 8, \mathrm{~S} 9, \mathrm{~S} 10, \mathrm{~S} 29, \mathrm{~T} 33, \mathrm{~S} 34, \mathrm{~S} 51, \mathrm{~S} 56$ \\
\hline SPTB2_HUMAN & SPTBN1 & $\mathrm{S} 2324$ & & $\mathrm{~T} 2328$ \\
\hline TPR_HUMAN & TPR & S1676 & & T1677 \\
\hline RBP2_HUMAN & RANBP2 & T1399 & T1399 & T1396, S1400 \\
\hline H31_HUMAN & HIST1H3A & S11 & S11 & $\mathrm{T} 12$ \\
\hline K2C8_HUMAN & KRT8 & $\mathrm{S} 13, \mathrm{~S} 15$ & S13 & S9, S13, T14 \\
\hline MYC_HUMAN & MYC & T58 & T58 & 562 \\
\hline NUMA1_HUMAN & NUMA1 & S1844 & & S1840, S1847 \\
\hline PHB_HUMAN & $\mathrm{PHB}$ & $\mathrm{T} 258$ & & S254 \\
\hline EMSY_HUMAN & EMSY & S236 & & S238 \\
\hline NU214_HUMAN & NUP214 & T1201, S1354 & & T1203, S1356 \\
\hline CRTC2_HUMAN & CRTC2 & $\mathrm{S} 70, \mathrm{~S} 171, \mathrm{~S} 173$ & S70, S171 & T169, S171, T177 \\
\hline KCC4_HUMAN & CAMK4 & S356 & S356 & S360 \\
\hline FOXO1_HUMAN & FOXO1 & T317 & & S319 \\
\hline BPTF_HUMAN & BPTF & T2094 & & S2098 \\
\hline HCFC1_HUMAN & HCFC1 & T738 & T738 & T737 \\
\hline K1C18_HUMAN & KRT18 & S30, S31, S49 & S30, S31 & S30, S31, S34, S47, S53 \\
\hline P121A_HUMAN & POM121 & T693 & & S697 \\
\hline RBM14_HUMAN & RBM14 & S244, S254, S256, S280 & $\mathrm{S} 256, \mathrm{~S} 280$ & S256 \\
\hline AKT1_HUMAN & AKT1 & T308, S473 & S473, T308 & \\
\hline ATX2L_HUMAN & ATXN2L & S684 & S684 & \\
\hline SYUA_HUMAN & SNCA & 587 & 587 & \\
\hline IKKB_HUMAN & IKBKB & S733 & S733 & \\
\hline ESR1_MOUSE & Esr1 & $\mathrm{S} 10$ & S10 & $\mathrm{T7}$ \\
\hline SPTB2_MOUSE & Sptbn1 & S2323 & & T2327 \\
\hline BSN_MOUSE & Bsn & S1407, S2027, S2029, T2700, T2703 & S2029, S2694, T2703 & T1406, S2029, T2703 \\
\hline SYN1_MOUSE & Syn1 & S518, T564 & & S520, S568 \\
\hline ABLM1_MOUSE & Ablim1 & S496, S499 & S496, S499 & S494, T495, S496, S499, S502 \\
\hline SKT_MOUSE & Skt & S357 & & S359, S361 \\
\hline DEMA_MOUSE & Epb49 & S285 & & S289 \\
\hline RBM14_MOUSE & Rbm14 & S278 & & S280 \\
\hline CEBPB_MOUSE & Cebpb & S180, S181 & & S184 \\
\hline SRBS1_MOUSE & Sorbs1 & S1199, S1200, S1201 & $\mathrm{S} 1201$ & S1201 \\
\hline ESR2_MOUSE & Esr2 & S61 & S61 & \\
\hline AKT1_MOUSE & Akt1 & S473 & S473 & \\
\hline NOS3_RAT & Nos3 & $\mathrm{S} 1178$ & & T1174, S1176 \\
\hline SP1_RAT & Sp1 & S613, T641, S642, S699, S703 & S613, T641, S642, S703 & T641, S642, S703 \\
\hline LBR_RAT & Lbr & 596 & & 599 \\
\hline TAU_RAT & Mapt & S711 & S711 & $\mathrm{S} 707, \mathrm{~T} 714, \mathrm{~S} 715$ \\
\hline KPCB_RAT & Prkcb & T635 & T635 & \\
\hline KPCD_RAT & Prkcd & T295, T348 & T295 & S299 \\
\hline KPCE_RAT & Prkce & $\mathrm{S} 368, \mathrm{~T} 710$ & $\mathrm{~S} 368, \mathrm{~T} 710$ & \\
\hline KPCG_RAT & Prkcg & T689, S690 & & S687 \\
\hline SYN1_RAT & Syn1 & S516, T562 & & S518, S566 \\
\hline G3P_RAT & Gapdh & T227 & T227 & \\
\hline LT_SV40 & SV40gp6 & $\mathrm{S} 111, \mathrm{~S} 112$ & $\mathrm{~S} 112$ & $\mathrm{~S} 112$ \\
\hline
\end{tabular}


Table 2 Major GO categories of human O-GIcNAcylated proteins

\begin{tabular}{|c|c|c|c|}
\hline Gene Ontology (GO) Terms & Count* & $\%$ Total & P-Value \\
\hline \multicolumn{4}{|l|}{ GO Biological Processes } \\
\hline GO:0045449 regulation of transcription & 108 & 23.48 & $5.90 \mathrm{E}-04$ \\
\hline GO:0006350 transcription & 93 & 20.22 & 1.99E-04 \\
\hline GO:0051252 regulation of RNA metabolic process & 75 & 16.30 & $6.09 \mathrm{E}-03$ \\
\hline GO:0006355 regulation of transcription, DNA-dependent & 69 & 15.00 & $3.08 \mathrm{E}-02$ \\
\hline GO:0043933 macromolecular complex subunit organization & 54 & 11.74 & 1.64E-09 \\
\hline GO:0065003 macromolecular complex assembly & 53 & 11.52 & $4.68 \mathrm{E}-10$ \\
\hline GO:0046907 intracellular transport & 52 & 11.30 & $8.96 \mathrm{E}-10$ \\
\hline GO:0007049 cell cycle & 52 & 11.30 & $2.14 \mathrm{E}-07$ \\
\hline GO:0006412 translation & 51 & 11.09 & $3.30 \mathrm{E}-21$ \\
\hline GO:0006396 RNA processing & 51 & 11.09 & $4.00 \mathrm{E}-12$ \\
\hline GO:0008104 protein localization & 51 & 11.09 & $1.91 \mathrm{E}-05$ \\
\hline GO:0010605 negative regulation of macromolecule metabolic process & 48 & 10.43 & $1.39 \mathrm{E}-06$ \\
\hline GO:0042981 regulation of apoptosis & 48 & 10.43 & $1.60 \mathrm{E}-05$ \\
\hline GO:0043067 regulation of programmed cell death & 48 & 10.43 & 2.07E-05 \\
\hline GO:0010941 regulation of cell death & 48 & 10.43 & $2.25 \mathrm{E}-05$ \\
\hline GO:0045184 establishment of protein localization & 47 & 10.22 & $1.11 \mathrm{E}-05$ \\
\hline GO:0010604 positive regulation of macromolecule metabolic process & 47 & 10.22 & $1.55 \mathrm{E}-04$ \\
\hline \multicolumn{4}{|l|}{ GO Molecular Function } \\
\hline GO:0000166 nucleotide binding & 132 & 28.70 & $2.56 \mathrm{E}-13$ \\
\hline GO:0003677 DNA binding & 101 & 21.96 & $7.11 \mathrm{E}-04$ \\
\hline GO:0032555 purine ribonucleotide binding & 92 & 20.00 & $7.02 \mathrm{E}-06$ \\
\hline GO:0032553 ribonucleotide binding & 92 & 20.00 & $7.02 \mathrm{E}-06$ \\
\hline GO:0017076 purine nucleotide binding & 92 & 20.00 & $3.91 \mathrm{E}-05$ \\
\hline GO:0030528 transcription regulator activity & 83 & 18.04 & $6.87 \mathrm{E}-07$ \\
\hline GO:0003723 RNA binding & 82 & 17.83 & $2.05 \mathrm{E}-24$ \\
\hline GO:0001882 nucleoside binding & 82 & 17.83 & $1.56 \mathrm{E}-05$ \\
\hline GO:0005524 ATP binding & 81 & 17.61 & $1.03 \mathrm{E}-06$ \\
\hline GO:0032559 adenyl ribonucleotide binding & 81 & 17.61 & $1.73 \mathrm{E}-06$ \\
\hline GO:0030554 adenyl nucleotide binding & 81 & 17.61 & $1.26 \mathrm{E}-05$ \\
\hline GO:0001883 purine nucleoside binding & 81 & 17.61 & $2.16 \mathrm{E}-05$ \\
\hline GO:0005198 structural molecule activity & 56 & 12.17 & $8.96 \mathrm{E}-12$ \\
\hline GO:0042802 identical protein binding & 51 & 11.09 & $3.27 \mathrm{E}-09$ \\
\hline \multicolumn{4}{|l|}{ GO Cellular Component } \\
\hline GO:0043232 intracellular non-membrane-bounded organelle & 170 & 36.96 & $6.37 \mathrm{E}-29$ \\
\hline GO:0043228 non-membrane-bounded organelle & 170 & 36.96 & $6.37 \mathrm{E}-29$ \\
\hline GO:0005829 cytosol & 141 & 30.65 & $7.16 \mathrm{E}-46$ \\
\hline GO:0031974 membrane-enclosed lumen & 131 & 28.48 & $6.60 \mathrm{E}-24$ \\
\hline GO:0043233 organelle lumen & 129 & 28.04 & $1.20 \mathrm{E}-23$ \\
\hline GO:0070013 intracellular organelle lumen & 128 & 27.83 & $5.00 \mathrm{E}-24$ \\
\hline GO:0031981 nuclear lumen & 116 & 25.22 & $2.44 \mathrm{E}-25$ \\
\hline GO:0005856 cytoskeleton & 77 & 16.74 & $2.21 \mathrm{E}-08$ \\
\hline GO:0005654 nucleoplasm & 77 & 16.74 & $2.07 \mathrm{E}-18$ \\
\hline GO:0030529 ribonucleoprotein complex & 72 & 15.65 & $3.55 \mathrm{E}-29$ \\
\hline GO:0005730 nucleolus & 55 & 11.96 & 3.09E-11 \\
\hline GO:0000267 cell fraction & 49 & 10.65 & $2.01 \mathrm{E}-03$ \\
\hline GO:0044430 cytoskeletal part & 49 & 10.65 & $1.16 \mathrm{E}-04$ \\
\hline
\end{tabular}

* The table is sorted based on counts of proteins annotated with given GO terms in each of the major GO category. The percentage of proteins with given GO terms over total number of human O-GIcNAcylated proteins is shown (\% Total). A more detailed GO profiles are shown in [Additional file 1, Supplementary Table S1]. 
Table 3 Pathway profiles using GeneGo Pathway Maps analysis

\begin{tabular}{|c|c|c|}
\hline Pathways & P-value & Count* \\
\hline Development_Role of CDK5 in neuronal development & $2.68 \mathrm{E}-11$ & $12 / 34$ \\
\hline Development_Gastrin in cell growth and proliferation & $3.86 \mathrm{E}-11$ & $15 / 62$ \\
\hline Immune response_Gastrin in inflammatory response & $2.00 \mathrm{E}-10$ & $15 / 69$ \\
\hline Signal transduction_Activation of PKC via G-Protein coupled receptor & $5.21 \mathrm{E}-10$ & $13 / 52$ \\
\hline Cytoskeleton remodeling_Cytoskeleton remodeling & $9.88 \mathrm{E}-10$ & $17 / 102$ \\
\hline Glycolysis and gluconeogenesis (short map) & 1.43E-09 & $14 / 67$ \\
\hline Cytoskeleton remodeling_Neurofilaments & $7.28 \mathrm{E}-09$ & $9 / 25$ \\
\hline Transcription_Role of Akt in hypoxia induced HIF1 activation & $1.59 \mathrm{E}-08$ & $9 / 27$ \\
\hline Immune response_MIF - the neuroendocrine-macrophage connector & $1.93 \mathrm{E}-08$ & $11 / 46$ \\
\hline Development_Prolactin receptor signaling & $2.50 \mathrm{E}-08$ & $12 / 58$ \\
\hline Cytoskeleton remodeling_TGF, WNT and cytoskeletal remodeling & $2.72 \mathrm{E}-08$ & $16 / 111$ \\
\hline Development_Gastrin in differentiation of the gastric mucosa & $3.22 \mathrm{E}-08$ & $10 / 38$ \\
\hline Development_GM-CSF signaling & 4.94E-08 & $11 / 50$ \\
\hline Development_EGFR signaling pathway & $6.66 \mathrm{E}-08$ & $12 / 63$ \\
\hline Cytoskeleton remodeling_Regulation of actin cytoskeleton by Rho GTPases & 7.06E-08 & $8 / 23$ \\
\hline G-protein signaling_Proinsulin C-peptide signaling & $7.62 \mathrm{E}-08$ & $11 / 52$ \\
\hline Development_Glucocorticoid receptor signaling & $1.04 \mathrm{E}-07$ & $8 / 24$ \\
\hline Development_VEGF signaling and activation & $1.16 \mathrm{E}-07$ & $10 / 43$ \\
\hline Cell adhesion_Histamine $\mathrm{H} 1$ receptor signaling in the interruption of cell barrier integrity & 1.85E-07 & $10 / 45$ \\
\hline Immune response_Inhibitory action of Lipoxins on pro-inflammatory TNF-alpha signaling & 1.85E-07 & $10 / 45$ \\
\hline Signal transduction_Calcium signaling & 1.85E-07 & $10 / 45$ \\
\hline Regulation of CFTR activity (norm and CF) & $2.50 \mathrm{E}-07$ & $11 / 58$ \\
\hline Translation_Regulation of translation initiation & $2.92 \mathrm{E}-07$ & $8 / 27$ \\
\hline Immune response_Histamine $\mathrm{H} 1$ receptor signaling in immune response & 3.53E-07 & $10 / 48$ \\
\hline Immune response_IL-2 activation and signaling pathway & 4.34E-07 & $10 / 49$ \\
\hline Transcription_P53 signaling pathway & $5.48 \mathrm{E}-07$ & $9 / 39$ \\
\hline Development_Slit-Robo signaling & 7.19E-07 & $8 / 30$ \\
\hline Development_Ligand-dependent activation of the ESR1/AP-1 pathway & $7.81 \mathrm{E}-07$ & $6 / 14$ \\
\hline Development_PDGF signaling via STATs and NF-kB & $1.24 \mathrm{E}-06$ & $8 / 32$ \\
\hline Signal transduction_AKT signaling & $1.33 \mathrm{E}-06$ & $9 / 43$ \\
\hline Development_VEGF signaling via VEGFR2 - generic cascades & $2.00 \mathrm{E}-06$ & $9 / 45$ \\
\hline Development_Thrombopoietin-regulated cell processes & $2.00 \mathrm{E}-06$ & 9/45 \\
\hline Mucin expression in CF via IL-6, IL-17 signaling pathways & 2.04E-06 & $8 / 34$ \\
\hline Development_TGF-beta-dependent induction of EMT via RhoA, PI3K and ILK. & 2.43E-06 & $9 / 46$ \\
\hline Development_PIP3 signaling in cardiac myocytes & 2.93E-06 & 9/47 \\
\hline Cytoskeleton remodeling_Keratin filaments & $3.24 \mathrm{E}-06$ & $8 / 36$ \\
\hline Development_Thyroliberin signaling & $3.61 \mathrm{E}-06$ & $10 / 61$ \\
\hline Development_A3 receptor signaling & $4.22 \mathrm{E}-06$ & 9/49 \\
\hline Transport_RAN regulation pathway & $4.42 \mathrm{E}-06$ & $6 / 18$ \\
\hline Immune response_IL-7 signaling in T lymphocytes & $5.01 \mathrm{E}-06$ & $8 / 38$ \\
\hline Muscle contraction_Regulation of eNOS activity in endothelial cells & $5.66 \mathrm{E}-06$ & $10 / 64$ \\
\hline Immune response_IL-6 signaling pathway & 7.57E-06 & $7 / 29$ \\
\hline
\end{tabular}

*Number of known O-GlcNAcylated proteins over the total number of proteins annotated in the corresponding GeneGO pathways. The table is ranked based on the pathway enrichment P-values. 
signaling (e.g., EGFR, VEGF, TGF $\beta$, and AKT) and cytokine signaling (e.g., GM-CSF, IL-2, IL6/7) pathways.

Because of the broad cellular processes and pathways that the O-GlcNAcylated proteins are known to participate in, O-GlcNAcylation may potentially play significant roles in many pathological conditions. Indeed, four categories of disease conditions have been implicated to involve O-GlcNAcylation, i.e., type II diabetes, neurodegenerative diseases, cardiovascular diseases, and cancers [34]. For example, OGT regulates insulin signaling through O-GlcNAcylation of several important insulin signaling molecules, e.g., IRS-1, PI3K, PDK1, and AKT1, leading to attenuation of insulin signaling responses in glycogen synthesis, activation of gluconeogenic genes and glucose transporter GLUT4 translocation, thus contributing to insulin resistance in type II diabetes $[1,35]$. Tau protein is subject to both OGlcNAcylation and phosphorylation, and hyperphosphorylation apparently contributes to neurofibrillary tangle formation by tau in Alzheimer's disease [36]. O-GlcNAcylation represents a key regulatory mechanism in modulating vascular reactivity, such as contractile and relaxant response through modification of proteins, e.g., NOS, sarcoplasmic reticulumn $\mathrm{Ca}(2+)$-ATPase, PKC, MAPK and cytoskeleton and microtubule proteins [37]. O-GlcNAcylation can mediate cardiac stress responses and has cardioprotective effects through transcription-mediated regulation as well as cardiomyocyte calcium homeostasis [38]. O-GlcNAcylation may have general roles in cancer through its involvement in oncogenesis or tumor suppression by coupling cellular metabolic status to regulation of signal transduction, transcription, and protein degradation. For example, reducing cellular UDP-GlcNAc level in MCF-7 cells changed the O-GlcNAcylation patterns of key proteins that control cell proliferation and differentiation, including Sp1, chaperonin TCP-1 theta, and oncogene Ets-related protein isoform 7 [39]. Many cancer genes or tumor suppressors are known to be O-GlcNAcylated or to interact with OGT, such as c-Myc, AKT1, AKT2, ATF1, CBP, FOXO1, TOP1, p53 and HIC1 [40]. In breast cancer cells, knockdown of OGT and the resulting global reduction of O-GlcNAcylation inhibited cell proliferation and metastasis ability [41].

\section{The O-GIcNAcylation Site Prediction}

Figure 3 (Above) shows the graphical representation of sequence patterns surrounding the O-GlcNAcylation sites annotated in dbOGAP using the "Two Sample Logo" tool [42]. Enrichment of amino acids at $-3 /+2$ position of the modified Ser/Thr, $\operatorname{PPV}(\underline{\mathrm{S} / \mathrm{T}}) \mathrm{TA}$, can be observed. However, the amino acid enrichment at each position independently is not sufficient for defining a sequence motif for O-GlcNAcylation sites. OGlcNAcScan was designed to exploit sequence properties through
SVM for the site prediction. The system achieved an area under $R O C$ (the receiver operating characteristic) curve (AUC) of 74.3\% (Figure 3, Below) in a five-fold cross-validation test. AUC is a widely used performance measure of binary classifiers. A perfect classifier yields an AUC of $100 \%$ while random guessing yields that of $50 \%$. Although the AUC value of OGlcNAcScan is relatively low, we need to consider at least the following two factors for its interpretation. First, the fraction of positive instances is extremely low in this task, i.e., 373 (1.23\%) of $30270 \mathrm{Ser} / \mathrm{Thr}$ sites are annotated O-GlcNAcylation sites in dbOGAP. Some of the past studies on PTM site prediction reported the performance of prediction systems on a balanced data set, where sampled negative sites were used in the evaluation data set (e.g., the ratio of positive and negative sites were made to be $1: 1$ (50\% positive) or $1: 5$ ( $16.7 \%$ positive)). In fact, the relative improvement of our trained SVM classifier, when compared to random guessing [43], can be as high as 14 -fold (i.e., the precision of the classifier can be 14 times higher than the original rate of positives sites of $1.23 \%)$. The second factor to be considered is that negative instances in the evaluation data set may include not-yet-annotated true O-GlcNAcylation sites, which could have lowered the performance measures. We believe, however, sequence-based prediction of OGlcNAcylation sites is inherently challenging. Additional training data through further annotation of proteins and sites as well as incorporation of other feature types, such as physiochemical properties of amino acids and protein structure information, may help improve the performance.

\section{The dbOGAP Web Site}

The dbOGAP web site provides two primary functionalities, search, query and browse of O-GlcNAcylated proteins and their related annotations, and de novo prediction of O-GlcNAcylation sites (Figure 4, \#1 and \#2). The dbOGAP database can be searched based on gene/protein names or identifiers, pathway names, or PubMed IDs. The protein entries can also be browsed based on gene names, organisms or pathways. The OGlcNAcScan site prediction system allows input of a protein sequence in FASTA format or a UniProtKB identifier (AC or ID) for site prediction. In addition, users can contribute their annotations to the database based on literature or from unpublished proteomic data on newly identified O-GlcNAcylation sites (Figure 4, \#3). All O-GlcNAcylation related literature citations are also available for browsing (Figure 4, \#4).

\section{The O-GIcNAcylated protein entry}

The dbOGAP protein entries are assigned unique IDs (e.g., OG00001) and are mapped to the corresponding UniProtKB IDs (1433B_HUMAN) and Accessions 


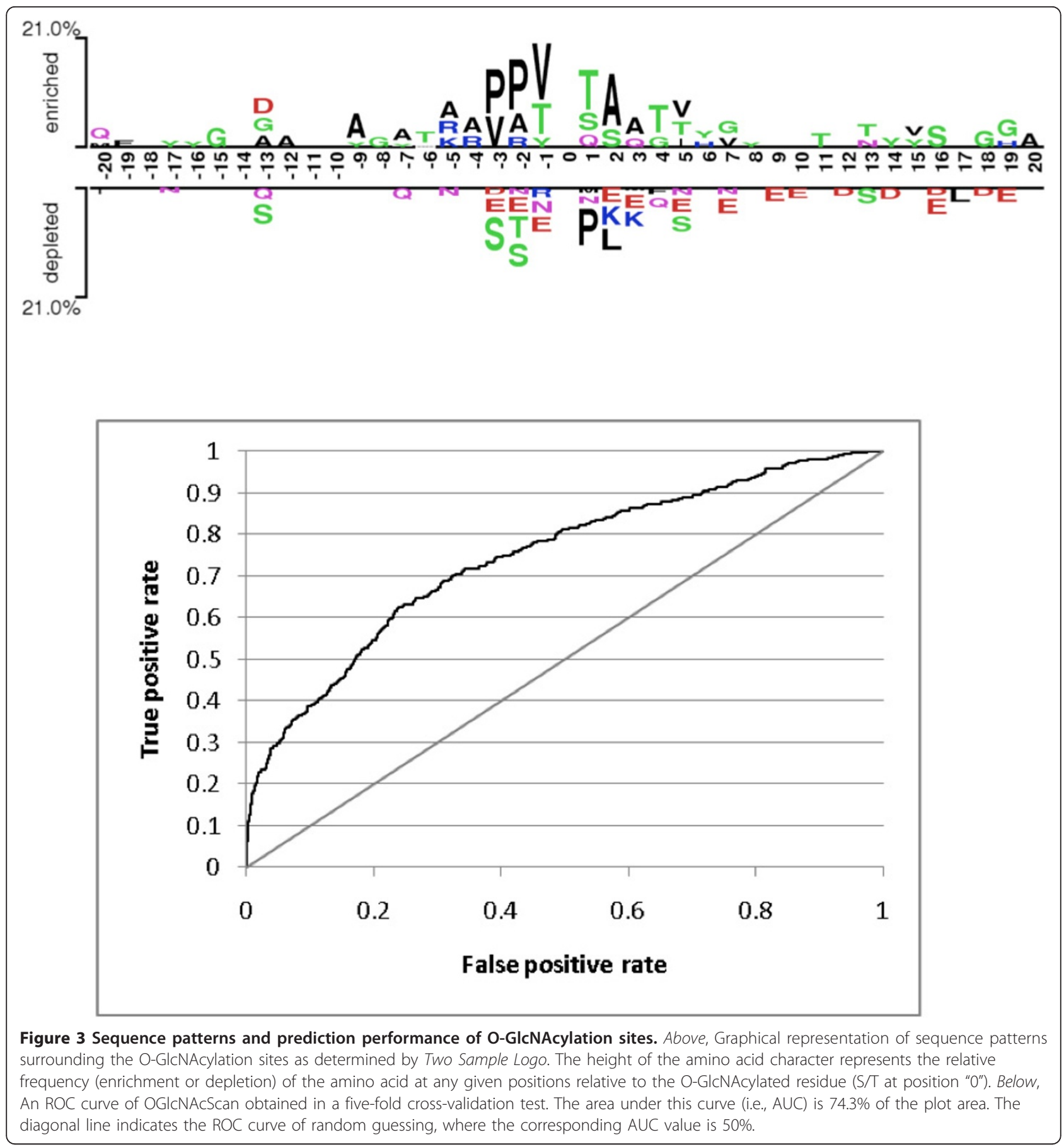

(P31946). The entry report provides detailed O-GlcNAcylation information and evidence attributions, including experimental and inferred O-GlcNAcylaytion data (Figure 5). O-GlcNAcylated residues and positions, as well as other modification sites (e.g., phosphorylation) and site features (e.g., binding sites), can be visualized in the context of protein sequences. The entry record also provides additional annotations such as GO, pathways (e.g., KEGG, PID and Reactome), protein-protein interactions (e.g., IntAct), protein families (e.g., Pfam) and diseases (OMIM), as well as additional protein bibliography integrated from UniProt and iProClass. Hyperlinks to source databases are provided for integrated annotations in dbOGAP entry records.

\section{The O-GIcNAcScan report}

The OGlcNAcScan report page provides a list of predicted O-GlcNAcylation sites for a given query sequence (Figure 6). The list can be sorted based on the 


\section{dbOGAP: Database of O-GIcNAcylated Proteins and Sites}

Georgetown|Lombardi

COMPREHENSIVE CANCER CENTER

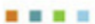

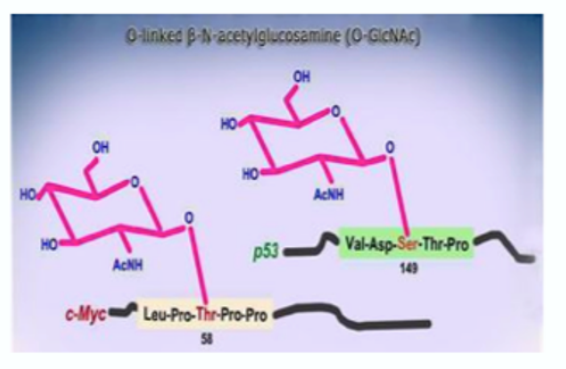

Introduction: Protein O-GIcNAcylation is an O-linked glycosylation involving attachment of beta- $N$ acetylglucosamine (GIcNAC) to Ser/Thr residues catalyzed by O-GlcNAc transferase (OGT) without further extension of GICNAC, whose removal is catalyzed by O-GICNAcase (OGA). Unlike N-linked and mucin-type O-linked glycosylation, O-GlcNAcylation occurs primarily in nucleocytoplasmic proteins, and is often dynamic and reciprocal to phosphorylation at the same or adjacent Ser/Thr residues (often mutually inhibitory). Compared to phosphorylation, the amount of research on 0-GIcNAcylation has been disproportionally small. Growing evidences now suggest that O-GlcNAcylation is common and has broad roles in physiology and diseases especially through its interplay with phosphorylation, e.g. regulation of insulin signaling and roles in diabetes and neurodegenerative diseases. To facilitate research on 0-GIcNAcylated proteins, we developed a database of O-GIcNAcylated proteins and sites (dbOGAP) based on experimental data curated from literature as well as from collaborating labs. The database also provides additional sequence annotations and functional information integrated from databases such as UniProt, and pathway and disease databases. Review statistics for the current version of dbOGAP (v1.0). For more, please see USHUPO 2010 abstract and presentation.

\section{1 Enter a term or ID: \\ Any Field : Search Reset \\ Browse database by: Gene Names A-E F-I J-N $0-R$ S-V W-Z | Organisms | Pathways | Download data sets | Statistics}

OGIcNAcScan, a Support Vector Machine-based sequence prediction

\section{2} program to predict protein 0-GIcNAcylation sites [Demo | Help]:

>sp|P01106 |MYC_BUMAN Myc proto-oncogene protein MPLNVSFTNRNYDLDYDSVPYFYCDEEENFYQQQQQSELQPPAPSEDIW KKPBLLPTPPLSP SRRSGLCSPSYVAVTPFSLRGDNDGGGSF STADQLB MVTELLGGDMVNQSFICDPDDETFIKNI IIQDCMWSGF SAAAKLVSEKLA SYQAARKDSGSPNPARGHSVCSTSSLYLQDLSAAASECIDP SVVFPYPLN DSSSPKSCASODSSAF SPSSDSLLSSTESSPOGSPEPLVLHEETPPTTSS DSEEEQEDEEEIDVVSVEKRQAPGKRSESGSPSAGGHSKPPHSPLVLKRC HVSTHOHNYAAPPSTRKDYPAAKRVKLDSVRVLROISNNRKCTSPRSSD? VENUKRRTEMYLERORRNEL KRSFPATRDOTPELEMNEKAPKYYILKKAT AYILSVQAEBQKLISEEDLLRKRREQLKHKLEQLRNSCA

Enter a protein UniProtAC e.g. P01106 or sequence in FASTA format

\section{Predict Reset}

Download the O-GICNAC sites data used to train the SVM model.

The discovery of the reciprocal relationship between protein O-GicNAcylation and phosphorylation represents a paradigm shift in studying the regulation of cell signaling. OGT and OGA interact closely with kinases, phosphatases and their respective substrates to form intricate networks of protein O-GIcNAcylation and phosphorylation in regulating cell signaling. Review references for more information.
You can help annotate the dbOGAP database!

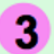

Annotate protein 0-GIcNAcyaltion [Help]:

You may enter any text here regarding the protein 0-GlcNAcyaltion and related information

Protein name* short, long, or synonym, e.g. p53

Protein ID/AC

Modified site

PubMed ID* e.g. 20016062; if not available, enter "NA"

Email address

* Required Submit Reset

Email address is not mandatory but desired for assuring the data quality Browse the 0-GICNAcylation Literature ( 490 0-GIcNAcylation related citations in the database)

Figure 4 The dbOGAP website home page. The website provides functionalities depicted by \#1-\#4: 1) search and browse the O-GlcNAcylated proteins in the database; 2) de novo prediction of O-GlcNAcylation sites for any protein sequences; 3) user annotation of O-GlcNAcylation information; 4) search and browse the total O-GlcNAcylation bibliography. The dbOGAP web site can be accessed at http://cbsb.lombardi. georgetown.edu/OGAP.html.

prediction scores, positions of predicted sites, and the amino acids. The predicted site of interest can be highlighted in the protein sequence. The threshold for display of O-GlcNAcylation sites can be adjusted to increase or reduce the number of predictions. More detailed explanation and interpretation of the prediction results are provided in the online help document.

\section{The dbOGAP database download}

The dbOGAP web site provides a download page (Figure 4 , linked in \#1) for downloading the database in several data files, including all O-GlcNAcylated proteins, sites and orthologs. A full bibliography of O-GlcNAcylated proteins can also be downloaded. The data sets for developing the OGlcNAcScan system are available to the scientific community for further development of the site prediction (Figure 4, \#2).

\section{Discussion}

Up to now, the amount of data published on protein $\mathrm{O}$ GlcNAcylation is only a fraction of that of phosphorylation, and its biological role is much less understood. Since 2006, the identification of O-GlcNAcylated proteins and sites has been rapidly growing due to the improved mass spectrometry technologies and O-GlcNAc enrichment 
techniques [7-9]. The dbOGAP database provides a timely bioinformatics resource to allow readily access by the community to the known and potential O-GlcNAcylated proteins and sites.

While a large number of O-GlcNAcylated proteins and sites were identified in recent years, many were determined based on large-scale mass spectrometry and would need to be further validated. Although O-
GlcNAcylation has been known to occur primarily in nucleocytoplamic proteins, the GO profiles show that $\mathrm{O}-\mathrm{GlcNA}$ cylated proteins are localized in a broad range of intracellular compartments. Interestingly, some OGlcNAcylated proteins are of unusual classes, e.g., adenylate kinase 2 (AK2, UniProtKB: KAD2_HUMAN) [44] localized in the mitochondria inter-membrane space, and alpha-1-inhibitor 3 (A1i3, UniProtKB: A1I3_RAT) 


\section{OGIcNAcScan results}

spIP16157|ANK1_HUMAN: Ankyrin-1 OS=Homo sapiens GN=ANK1 PE=1 SV=3

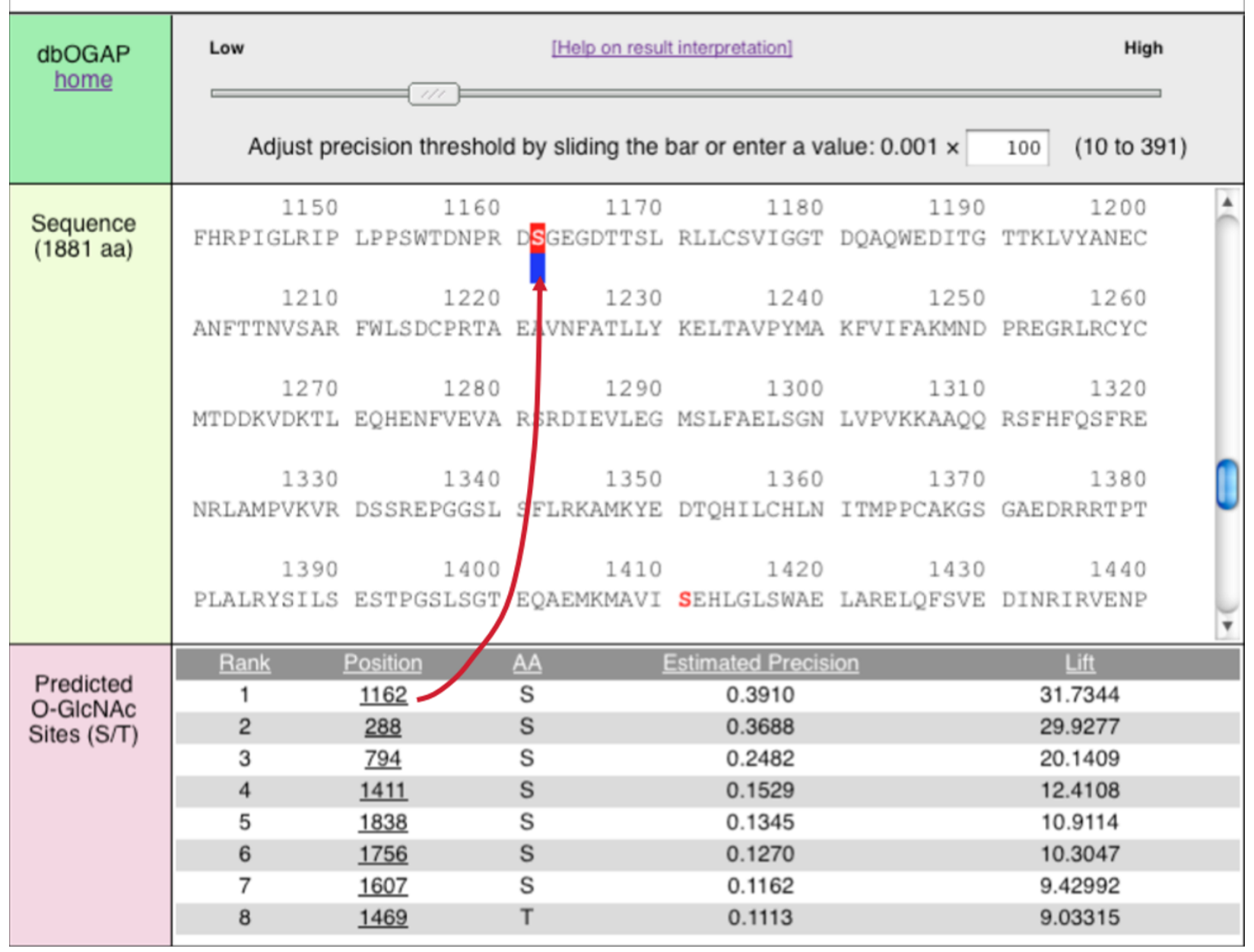

Figure 6 The O-GIcNAcylation site prediction result from OGIcNAcScan (shown is human ankyrin-1). The section at the bottom displays a ranked list of predicted O-GlcNAcylation sites (e.g., S1162 as the top one). The rank is based on the output value of the SVM classifier, which is converted into "Estimated Precision" and "Lift" scores (see help page linked from the top of the page for explanation). The estimated precision score is an estimated lower-bound of the precision (e.g., the score of 0.3910 indicates that at least $39.1 \%$ of sites assigned with the similar SVM output scores are O-GlcNAcylation sites), and the Lift score is an index of relative improvement through the classifier, which is calculated as the estimated precision divided by a constant value corresponding to the initial rate of positive sites (i.e., $\sim 0.0123$ ). All displayed potential sites are shown as red "S/T" in the sequence section (middle). Clicking on any predicted site, the residue will be highlighted in the sequence (arrow).

[45], a secreted protein. Although false positive identification of O-GlcNAcylation is not uncommon from mass spectrometry, it is possible that such proteins may be indeed O-GlcNAcylated. It is known that OGT has at least three isoforms differing in $\mathrm{N}$-terminal sequences with identical catalytic domain, the mitochondrial (mOGT) and two nucleocytoplasmic forms (ncOGT and sOGT) $[46,47]$. The mOGT form was shown associated with the mitochondrial inner membrane [46], thus consistent with the observation of O-GlcNAcylation of the mitochondrial protein AK2. There are a total of $\sim 11 \mathrm{O}-$ GlcNAcylated proteins in dbOGAP that are known to be secreted or have secreted forms besides cytoplasmic ones. It is possible that only the cytoplasmic forms of some of these proteins are O-GlcNAcylated while the secreted ones may not, albeit experimental validation is needed. Thus, the types and/or sources of O-GlcNAcylation identification have been assigned to protein entries as evidence attribution to annotations in the dbOGAP database.

The OGlcNAcScan site prediction system provides a much needed tool for studying protein glycosylation as well as phosphorylation. Since the site prediction is primarily based on the protein sequence context, some 
secreted proteins may be erroneously predicted even with a relatively high score, e.g., T298 in mucin 4 (UniProtKB: MUC4_HUMAN) predicted with a score of 0.287 , though it is unlikely to be O-GlcNAcylated. In such cases, a cautionary note is given to indicate that a protein sequence being predicted is known to have "secreted" form(s). With the continuing growth of O-GlcNAcylation sites data, the OGlcNAcScan tool will be further enhanced through retraining the SVM model, as well as by integrating physiochemical properties and structural information into the SVM prediction model.

\section{Conclusion}

In conclusion, the dbOGAP database and the web site become the first of its kind in the public domain to provide readily access to a curated and systematic collection of protein O-GlcNAcylation information, and to a stateof-the-art O-GlcNAcylation site prediction system, OGlcNAcScan, to assist proteomic identification of OGlcNAc modification sites. Thus, the dbOGAP resource should benefit the biological community to study the broad roles of O-GlcNAcylation in physiology and diseases.

\section{Availability and Requirements}

The dbOGAP database and the OGlcNAcScan system can be publicly accessed at: http://cbsb.lombardi.georgetown.edu/OGAP.html. The database and related data sets can be downloaded at: http://cbsb.lombardi.georgetown.edu/filedown.php.

\section{Additional material}

Additional file 1: Supplementary Table S1. Major categories of OGIcNAcylated proteins based on GO terms at deeper level of GO hierarchy. This table provides $\mathrm{GO}$ profiles at deeper level of $\mathrm{GO}$ terms to complement the major GO profiles of O-GlcNAcylated proteins in Table 2.

\section{Acknowledgements}

We would like to acknowledge the support from Lombardi Comprehensive Cancer Center (LCCC) at Georgetown University Medical Center. J.W. was supported by a postdoctoral fellowship at the LCCC, and M.T., H.L and Z.Z.H are partially supported by NIH/NLM grant 1R01LM009959-01A1. We also would like to thank Jinesh Shah for assisting curation of evidence attributions reported in literature for O-GlcNAcylation data from large-scale mass spectrometry.

\section{Author details}

'Department of Oncology, Georgetown University Medical Center, 3300 Whitehaven Street, Suite 1200, NW, Washington, DC 20007, USA. 'ISIS Center, Georgetown University Medical Center, 2115 Wisconsin Avenue, Suite 603, Washington, DC 20007, USA. ${ }^{3}$ Department of Biostatistics, Bioinformatics and Biomathematics, Georgetown University Medical Center, 4000 Reservoir Road, Building D, Suite 180, NW, Washington, DC 20057, USA. ${ }^{4}$ Biological Chemistry, School of Medicine, Johns Hopkins University, 725 N. Wolfe Street, 515 WBSB, Baltimore, MD 21205, USA.

\section{Authors' contributions}

JW is responsible for the design and implementation of the database and the web site development for most of the web pages. MT is responsible for developing the OGICNAcScan system and the web display of the prediction results. HL contributed to the machine learning methods for OGICNAcScan and to the design and testing of the dbOGAP web interface. GWH contributed experimental O-GlcNAcylation data for populating the database. $\mathrm{ZZH}$ conceived the overall design of the database and site prediction, and is responsible for the O-GlcNAcylation data curation from literature and the web site testing. All authors read and approved the manuscript.

Received: 4 November 2010 Accepted: 6 April 2011

Published: 6 April 2011

\section{References}

1. Hart GW, Housley MP, Slawson C: Cycling of O-linked beta-Nacetylglucosamine on nucleocytoplasmic proteins. Nature 2007, 446:1017-1022.

2. Copeland RJ, Bullen JW, Hart GW: Cross-talk between GlcNAcylation and phosphorylation: roles in insulin resistance and glucose toxicity. Am J Physiol Endocrinol Metab 2008, 295:E17-28.

3. Wang Z, Gucek M, Hart GW: Cross-talk between GlcNAcylation and phosphorylation: site-specific phosphorylation dynamics in response to globally elevated O-GIcNAc. Proc Natl Acad Sci USA 2008, 105:13793-13798.

4. Wells L, Kreppel LK, Comer FI, Wadzinski BE, Hart GW: O-GICNAc transferase is in a functional complex with protein phosphatase 1 catalytic subunits. J Biol Chem 2004, 279:38466-38470.

5. Cheng X, Hart GW: Alternative O-glycosylation/O-phosphorylation of serine-16 in murine estrogen receptor beta: post-translational regulation of turnover and transactivation activity. J Biol Chem 2001, 276:10570-5.

6. Yang WH, Kim JE, Nam HW, Ju JW, Kim HS, Kim YS, Cho JW: Modification of p53 with O-linked $\mathrm{N}$-acetylglucosamine regulates p53 activity and stability. Nat Cell Biol 2006, 8:1074-1083.

7. Wang Z, Udeshi ND, O'Malley M, Shabanowitz J, Hunt DF, Hart GW: Enrichment and site-mapping of O-Linked N-Acetylglucosamine by a combination of chemical/enzymatic tagging, photochemical cleavage, and electron transfer dissociation (ETD) mass spectrometry. Mol Cell Proteomics 2009, 9:153-160.

8. Torres $\mathrm{CR}$, Hart GW: Topography and polypeptide distribution of terminal $\mathrm{N}$-acetylglucosamine residues on the surfaces of intact lymphocytes. Evidence for O-linked GIcNAc. J Biol Chem 1984, 259:3308-3317.

9. Vosseller K, Trinidad JC, Chalkley RJ, Specht CG, Thalhammer A, Lynn AJ, Snedecor JO, Guan S, Medzihradszky KF, Maltby DA, Schoepfer R, Burlingame AL: O-linked N-acetylglucosamine proteomics of postsynaptic density preparations using lectin weak affinity chromatography and mass spectrometry. Mol Cell Proteomics 2006, 5:923-934.

10. Nandi A, Sprung R, Barma DK, Zhao Y, Kim SC, Falck JR, Zhao Y: Global identification of O-GICNAc-modified proteins. Anal Chem 2006, 78:452-458.

11. Khidekel N, Ficarro SB, Clark PM, Bryan MC, Swaney DL, Rexach JE, Sun YE, Coon JJ, Peters EC, Hsieh-Wilson LC: Probing the dynamics of O-GIcNAC glycosylation in the brain using quantitative proteomics. Nat Chem Biol 2007, 3:339-348

12. Lee TY, Huang HD, Hung JH, Huang HY, Yang YS, Wang TH: dbPTM: an information repository of protein post-translational modification. Nucleic Acids Res 2006, , 34 Database: D622-627.

13. Diella F, Cameron S, Gemünd C, Linding R, Via A, Kuster B, SicheritzPontén T, Blom N, Gibson TJ: Phospho.ELM: A database of experimentally verified phosphorylation sites in eukaryotic proteins. BMC Bioinformatics 2004, 5:79.

14. Hornbeck PV, Chabra I, Kornhauser JM, Skrzypek E, Zhang B: PhosphoSite: A bioinformatics resource dedicated to physiological protein phosphorylation. Proteomics 2004, 4:1551-1561.

15. Gupta R, Birch H, Rapacki K, Brunak S, Hansen JE: O-GLYCBASE version 4.0: a revised database of O-glycosylated proteins. Nucleic Acids Res 1999, 27:370-372.

16. Chernorudskiy AL, Garcia A, Eremin EV, Shorina AS, Kondratieva EV, Gainullin MR: UbiProt: a database of ubiquitylated proteins. BMC Bioinformatics 2007, 8:126.

17. Rawlings ND, Barrett AJ, Bateman A: MEROPS: the peptidase database. Nucleic Acids Res 2010, , 38 Database: D227-233. 
18. UniProt Consortium: The Universal Protein Resource (UniProt) in 2010. Nucleic Acids Res 2010, , 38 Database: D142-148.

19. Consortium for Functional Glycomics (CFG). [http://www. functionalglycomics.org/].

20. Hansen JE, Lund O, Tolstrup N, Gooley AA, Williams KL, Brunak S: NetOglyc: prediction of mucin type O-glycosylation sites based on sequence context and surface accessibility. Glycoconj J 1998, 15:115-130.

21. Julenius K, Mølgaard A, Gupta R, Brunak S: Prediction, conservation analysis, and structural characterization of mammalian mucin-type Oglycosylation sites. Glycobiology 2005, 15:153-164.

22. Chen YZ, Tang YR, Sheng ZY, Zhang Z: Prediction of mucin-type Oglycosylation sites in mammalian proteins using the composition of kspaced amino acid pairs. BMC Bioinformatics 2008, 9:101

23. Torii M, Liu H, Hu ZZ: Support vector machine-based mucin-type Oglycosylation site prediction using enhanced sequence feature encoding. AMIA Annu Symp Proc 2009, 640-644.

24. Gupta R, Brunak S: Prediction of glycosylation across the human proteome and the correlation to protein function. Pacific Symposium on Biocomputing 2002, 310-322.

25. HomoloGene. [http://www.ncbi.nlm.nih.gov/homologene].

26. PIR Blast neighbors. [http://pir.georgetown.edu/pirwww/search/].

27. Wu CH, Huang H, Nikolskaya A, Hu Z, Barker WC: The iProClass integrated database for protein functional analysis. Comput Biol Chem 2004, 28:87-96.

28. Joachims T: Text Categorization with Support Vector Machines: Learning with Many Relevant Features. European Conference on Machine Learning 1998, 137-142

29. Huang da W, Sherman BT, Tan Q, Kir J, Liu D, Bryant D, Guo Y, Stephens R, Baseler MW, Lane HC, Lempicki RA: DAVID Bioinformatics Resources: expanded annotation database and novel algorithms to better extract biology from large gene lists. Nucleic Acids Res 2007, , 35 Web Server: W169-75.

30. Buse MG, Robinson KA, Marshall BA, Hresko RC, Mueckler MM: Enhanced OGlcNAc protein modification is associated with insulin resistance in GLUT1-overexpressing muscles. Am J Physiol Endocrinol Metab 2002, 283 E241-250.

31. Park SY, Ryu J, Lee W: O-GIcNAc modification on IRS-1 and Akt2 by PUGNAc inhibits their phosphorylation and induces insulin resistance in rat primary adipocytes. Exp Mol Med 2005, 37:220-229.

32. Matsuura A, Ito M, Sakaidani $Y$, Kondo T, Murakami K, Furukawa $\mathrm{K}$, Nadano D, Matsuda T, Okajima T: O-linked N-acetylglucosamine is present on the extracellular domain of notch receptors. J Biol Chem 2008, 283:35486-35495.

33. Ekins S, Nikolsky Y, Bugrim A, Kirillov E, Nikolskaya T: Pathway mapping tools for analysis of high content data. Methods Mol Biol 2007, 356:319-350.

34. Slawson C, Copeland RJ, Hart GW: O-GICNAc signaling: a metabolic link between diabetes and cancer? Trends Biochem Sci 2010, 35:547-555.

35. Yang X, Ongusaha PP, Miles PD, Havstad JC, Zhang F, So WV, Kudlow JE, Michell RH, Olefsky JM, Field SJ, Evans RM: Phosphoinositide signalling links O-GICNAc transferase to insulin resistance. Nature 2008, 451:964-969.

36. Liu F, Shi J, Tanimukai H, Gu J, Gu J, Grundke-lqbal I, Iqbal K, Gong CX: Reduced O-GIcNAcylation links lower brain glucose metabolism and tau pathology in Alzheimer's disease. Brain 2009, 132:1820-1832.

37. Lima W, Rigsby CS, Hardy DM, Webb RC, Tostes RC: O-GlcNAcylation: a novel post-translational mechanism to alter vascular cellular signaling in health and disease: focus on hypertension. J Am Soc Hypertens 2009, 3:374-387.

38. Chatham JC, Marchase RB: The role of protein O-linked beta-Nacetylglucosamine in mediating cardiac stress responses. Biochim Biophys Acta 2010, 1800:57-66.

39. Lefebvre T, Pinte S, Guérardel C, Deltour S, Martin-Soudant N, Slomianny MC, Michalski JC, Leprince D: The tumor suppressor HIC1 (hypermethylated in cancer 1) is O-GlcNAc glycosylated. Eur J Biochem 2004, 271:3843-3854

40. Donadio AC, Lobo C, Tosina M, de la Rosa V, Martín-Rufián M, CamposSandoval JA, Matés JM, Márquez J, Alonso FJ, Segura JA: Antisense glutaminase inhibition modifies the O-GlcNAc pattern and flux through the hexosamine pathway in breast cancer cells. J Cell Biochem 2008, 103:800-811.
41. Caldwell SA, Jackson SR, Shahriari KS, Lynch TP, Sethi G, Walker S, Vosseller K, Reginato MJ: Nutrient sensor O-GICNAc transferase regulates breast cancer tumorigenesis through targeting of the oncogenic transcription factor FoxM1. Oncogene 2010, 29:2831-2842.

42. Vacic V, lakoucheva LM, Radivojac P: Two Sample Logo: a graphical representation of the differences between two sets of sequence alignments. Bioinformatics 2006, 22:1536-1537.

43. Coppock DS: Why Lift? Data Modeling and Mining. Information Management Online 2002 [http://www.information-management.com/news/ 5329-1.html], Last visited October 11, 2010.

44. Nandi A, Sprung R, Barma DK, Zhao Y, Kim SC, Falck JR, Zhao Y: Global identification of O-GlcNAc-modified proteins. Anal Chem 2006, 78:452-458.

45. Cieniewski-Bernard C, Bastide B, Lefebvre T, Lemoine J, Mounier Y, Michalski JC: Identification of O-linked $\mathrm{N}$-acetylglucosamine proteins in rat skeletal muscle using two-dimensional gel electrophoresis and mass spectrometry. Mol Cell Proteomics 2004, 3:577-585.

46. Love DC, Kochan J, Cathey RL, Shin SH, Hanover JA: Mitochondrial and nucleocytoplasmic targeting of O-linked GlcNAc transferase. J Cell Sci 2003, 116(Pt 4):647-654.

47. Lazarus BD, Love DC, Hanover JA: Recombinant O-GIcNAc transferase isoforms: identification of O-GIcNAcase, yes tyrosine kinase, and tau as isoform-specific substrates. Glycobiology 2006, 16:415-421.

\section{doi:10.1186/1471-2105-12-91}

Cite this article as: Wang et al.: dbOGAP - An Integrated Bioinformatics Resource for Protein O-GlcNAcylation. BMC Bioinformatics 2011 12:91.

\section{Submit your next manuscript to BioMed Central and take full advantage of:}

- Convenient online submission

- Thorough peer review

- No space constraints or color figure charges

- Immediate publication on acceptance

- Inclusion in PubMed, CAS, Scopus and Google Scholar

- Research which is freely available for redistribution

Submit your manuscript at www.biomedcentral.com/submit
C Biomed Central 